\title{
The effect of plume/interplume lanes on ion-cyclotron resonance heating
}

\author{
Suzan Doğan and E. Rennan Pekünlü \\ Department of Astronomy and Space Sciences, Faculty of Science, University of Ege, Bornova, \\ 35100, İzmir, Turkey \\ email: dogansuzan@gmail.com \\ email: rennan.pekunlu@ege.edu.tr
}

\begin{abstract}
The effect of the Plume/Interplume Lane (PIPL) structure of the solar North Polar Coronal Hole (NPCH) on the ion-cyclotron resonance (ICR) process is investigated. The ICR process in the interplume lanes is much more effective than in the plumes, agreeing with the observations which show the source of fast solar wind is interplume lanes.
\end{abstract}

Keywords. Sun: corona, solar wind, acceleration of particles, plasmas, waves.

\section{Introduction}

Images taken by SOHO/SUMER clearly show that the solar NPCH is structured in radial direction by so-called plume and interplume lanes (PIPL) (see Fig. 1). Plumes are denser and cooler, interplumes are less dense and hotter regions.

Doppler dimming analysis (Kohl et al. 1997) showed that the O VI ions display a temperature anisotropy with $T_{\perp} \sim 10^{2} T_{\|}$. Besides, outflow velocities in the solar wind of $\mathrm{O}$ VI ions in the interplume lanes are higher than that of the plumes. The origin of the fast solar wind was identified as the interplume lanes (Wilhelm et al. 1998; Hollweg 1999a, 1999b, 1999c). These effects could be brought about only by ICR process (Hollweg 1999a, 1999b, 1999c). Therefore, ICR process is regarded as the most efficient heating mechanism for NPCH.

The aim of this study is to investigate the effect of the PIPL structure of the solar $\mathrm{NPCH}$ on the propagation characteristics of ion-cyclotron waves (ICW). The gradients of physical parameters both parallel and perpendicular to the magnetic field are considered with the aim of determining how the efficiency of the ICR process varies along the PIPL structure of NPCH.

\section{Model}

Our model is based on the kinetic theory. We solve the Vlasov equation for O VI ions and obtain the dispersion relation of ICW. O VI ions are considered under the two forces, a) Lorentz force, b) pressure gradient force. The quasi-linearized Vlasov equation is:

$$
\begin{aligned}
& \frac{d f_{1}}{d t}=\frac{\partial f_{1}}{\partial t}+\mathbf{v} \cdot \frac{\partial f_{1}}{\partial \mathbf{R}}+\frac{q_{\mathrm{i}}}{m_{\mathrm{i}}}\left(\mathbf{E}_{1}+\frac{1}{c}\left(\mathbf{v} \times \mathbf{B}_{0}\right)\right) \cdot \frac{\partial f_{1}}{\partial \mathbf{v}}=\left[-\frac{q_{\mathrm{i}}}{m_{\mathrm{i}}}\left(\mathbf{E}_{1}+\frac{1}{c}\left(\mathbf{v} \times \mathbf{B}_{1}\right)\right)\right. \\
& \left.+\frac{k_{\mathrm{B}}}{n_{0} m_{\mathrm{i}}}\left(T_{\text {eff }}^{\xi} \frac{\partial n_{0}}{\partial \mathbf{R}}+T_{\text {eff }}^{\xi} \frac{\partial n_{0}}{\partial \mathbf{x}}+n_{0} \frac{\partial T_{\text {eff }}^{\xi}}{\partial \mathbf{R}}+n_{0} \frac{\partial T_{\text {eff }}^{\xi}}{\partial \mathbf{x}}\right)\right] \cdot \frac{\partial f_{0}}{\partial \mathbf{v}}
\end{aligned}
$$




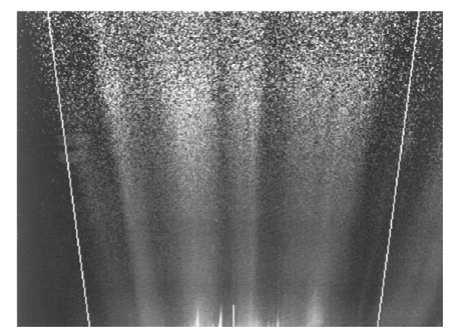

Figure 1. SOHO/SUMER image of polar plumes and interplumes in O VI (1032 A). This image was obtained over the North pole in 1996 (Wilhelm et al. 1998).

where $f_{0}$ and $f_{1}$ are the unperturbed and perturbed parts of the velocity distribution function, and $\mathbf{E}_{1}$ and $\mathbf{B}_{1}$ are the wave electric and magnetic fields, respectively.

$$
f_{0}=n_{\mathrm{i}} \alpha_{\perp}^{2} \alpha_{\|} \pi^{-3 / 2} \exp \left[-\left(\alpha_{\perp}^{2} v_{\perp}^{2}+\alpha_{\|}^{2} v_{\|}^{2}\right)\right]
$$

where $\alpha_{\perp}=\left(2 k_{\mathrm{B}} T_{\perp} / m_{i}\right)^{-1 / 2}$ and $\alpha_{\|}=\left(2 k_{\mathrm{B}} T_{\|} / m_{i}\right)^{-1 / 2}$ are the inverse of the most probable speeds in the perpendicular and parallel direction to the external magnetic field, respectively. By solving the Vlasov equation analytically and neglecting the residual contribution, we find the dispersion relation of ICW as below,

$$
k^{2}\left[c^{2}-\frac{i \omega_{p}^{2}}{2 \alpha_{\|}^{2}\left(\omega-\omega_{c}\right)^{2}} \Pi\right]-\frac{i \omega_{p}^{2} k}{\sqrt{\pi} \alpha_{\|}\left(\omega-\omega_{c}\right)} \Pi-\frac{i \omega_{p}^{2} \omega}{\left(\omega-\omega_{c}\right)}-\omega^{2}+\frac{\pi^{2} \omega q_{\mathrm{i}} L}{E_{x} v_{A}} \nabla p_{1}^{\mathrm{r}}=0
$$

where $\omega_{p}$ is the plasma frequency, $\omega_{c}$ is the cyclotron frequency for the O VI ions. For the sake of brevity we replace $\left[\omega /\left(\omega-\omega_{c}\right)\right]+\left(T_{\perp} / T_{\|}\right)-1$ by $\Pi$ and $\nabla p_{1}^{r}$ is the term designating the pressure gradient:

$$
\nabla p_{1}^{\mathrm{r}}=\frac{k_{\mathrm{B}}}{m_{\mathrm{i}}}\left[\left(T_{\mathrm{eff}}^{\xi} \frac{\partial n_{0}}{\partial R}+n_{0} \frac{\partial T_{\mathrm{eff}}^{\xi}}{\partial R}\right)\left(\frac{T_{\perp}}{T_{\|}}\right)^{1 / 2}+2\left(T_{\mathrm{eff}}^{\xi} \frac{\partial n_{0}}{\partial x}+n_{0} \frac{\partial T_{\mathrm{eff}}^{\xi}}{\partial x}\right)\right]
$$

\section{Results}

Dispersion relation of the ICW given by Eq. (2.3) is solved for two situations, $(i)$ the PIPL structure of NPCH is not taken into account, $(i i)$ the PIPL structure therefore the plasma gradients in $x$ direction are taken into account. These solutions are compared in Fig. 2. For $\omega=2500 \mathrm{rads}^{-1}$, all the solutions reveal an infinity in the refractive index, corresponding to a resonance at about $2.38 \mathrm{R}$. We should emphasize that the waves with frequencies higher than $2500 \mathrm{rads}^{-1}$ resonate with O VI ions at smaller $\mathrm{R}$ and vice versa.

When the PIPL structure is considered, the differences in the refractive index of the plumes and interplume lanes also revealed. Fig. $2 \mathrm{~b}$ and $2 \mathrm{~d}$ show crests which correspond to the interplume lanes and troughs which correspond to the plumes. As it is apparent from the figures that refractive index in the interplume lanes are about 2 times higher than the ones in the plumes. We may argue that the refractive index of the interplume lanes is readily going to infinity indicating that the resonance process in the interplume lanes is more effective than in the plumes. 

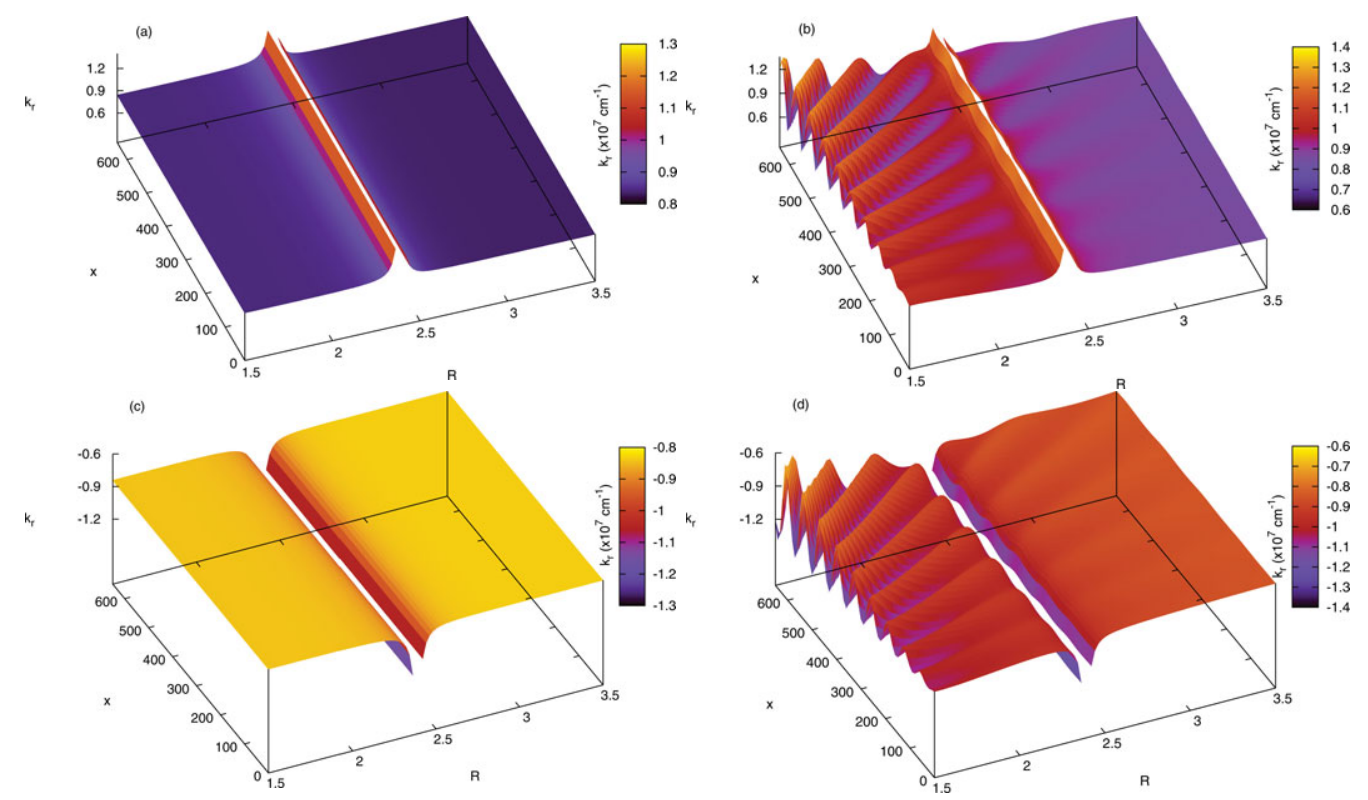

Figure 2. Two roots of the second order dispersion relation given by Eq. (2.3). $\mathrm{R}$ is the dimensionless radial distance $\left(r / R_{\odot}\right)$ and $x$ is the distance perpendicular to R. (a) Forward propagating mode when PIPL structure is not taken into account; (b) the same mode with PIPL structure considered; (c) backward propagating mode without PIPL structure and (d) the same mode with PIPL structure considered. The graphs are for ICW with a frequency $2500 \mathrm{rads}^{-1}$.

When we adopt $10^{-3}$ for $N_{O V I} / N_{p}$, which is the value Vocks (2002) used in his model, the ratio of the wave numbers $k_{\nabla p} / k$ range from 0.2 to 16.8 in the distance range $1.5-3.5$ $\mathrm{R}$, where $k_{\nabla p}$ is the wave number of the waves propagating in the presence of gradients considered. For $N_{O V I} / N_{p}=1.52 \times 10^{-6}$ which is the value given by Cranmer et al. (2008), $k_{\nabla p} / k$ take values between 0.7 and 1.6 . We plotted the figures by adopting the later value, i.e. Cranmer et al.'s (2008).

\section{Conclusion}

When the wave frequency is equal to the ion cyclotron frequency, i.e., $\omega=\omega_{c}$, ICW resonate with and transfer their energy to the $\mathrm{O}$ VI ions. The resonance process in the interplume lanes is more effective than in the plumes. This result is confirmed by the observations showing that the seat of the fast solar wind is interplume lanes.

\section{References}

Cranmer, S. R., Panasyuk, A. V., \& Kohl, J. L. 2008, ApJ, 678, 1480

Hollweg, J. V. 1999a, JGR, 104, 24781

Hollweg, J. V. 1999b, JGR, 104, 24793

Hollweg, J. V. 1999c, JGR, 104, 505

Kohl, J. L., Noci, G., Antonucci, E. et al. 1997, SoPh, 175, 613

Wilhelm, K., Marsch, E., Dwivedi, B. N. et al. 1998, ApJ, 500, 1023 